Check for updates

Cite this: Analyst, 2020, 145, 6079

\section{A SERS-based competitive immunoassay for highly sensitive and specific detection of ochratoxin A}

\begin{abstract}
Yangjun Ding, ${ }^{a}$ Hezhen Shang, ${ }^{b}$ Xiaokun Wang ${ }^{\star c}$ and Lingxin Chen (D)*d,e
Ochratoxin A (OTA) is a well-known carcinogenic contaminant in food commodities. The highly sensitive and specific detection of the OTA level is an essential way to prevent the health risks to humans/animals. In this work, an SERS-based competitive immunoassay platform has been developed for the detection of OTA. This assay contains two compartments: OTA-BSA-immobilized SERS nanotags and anti-OTA antibody-functionalized magnetic beads. In the presence of target OTA, a competitive reaction towards magnetic beads occurs between the target OTA and SERS nanotags. The characteristic peaks of SERS nanotags adsorbed onto magnetic beads were used for the quantitative analysis of OTA. Under optimized conditions, a good linear relationship was obtained in the range of $1 \mathrm{pg} \mathrm{mL}^{-1}$ to1000 $\mathrm{pg} \mathrm{mL}^{-1}$. The limit of detection (LOD) was estimated to be $0.61 \mathrm{pg} \mathrm{mL}^{-1}$ using the IUPAC standard method. In addition, this proposed method was also successfully applied for the detection of OTA in wine samples and satisfactory recovery rates were obtained in the range of $90.60 \%$ to $103.3 \%$. It is believed that this SERS-based competitive method holds great potential for highly sensitive and specific detection of mycotoxins.
\end{abstract}

Received 18th June 2020 Accepted 25th July 2020 DOI: $10.1039 / \mathrm{dOan} 01220 \mathrm{c}$ rsc.li/analyst
OTA and other types of mycotoxins in food and feed commodities. To ensure food safety, it is very urgent to develop highly sensitive and specific detection methods for monitoring even trace amounts of OTA in food systems.

To date, many techniques have been used to develop a reliable analysis method for the identification of OTA, including chromatography techniques and enzyme-linked immunosorbent assay (ELISA). Chromatography techniques, such as high-performance liquid chromatography (HPLC), gas chromatography-mass spectrometry (GC-MS), and liquid chromatography-mass spectrometry (LC-MS) have a satisfactory limit of detection. $^{8-11}$ However, these methods require expensive equipment, skilled operators, and multiple operation steps, which are time-consuming and unsuitable for routine analysis applications. Considering the high reliability and reproducibility, ELISA using a 96-well plate has become one of the most widely used immunoassay methods in biological laboratories, and various commercialized ELISA kits have been developed for the determination of OTA. However, the enzyme-mediated color change at low concentrations of the target cannot be identified by the microplate reader. Thus, ELISA alone is not sufficient to sensitively detect OTA. ${ }^{12}$ To overcome these shortcomings, a variety of transduction methods have been integrated into ELISA to improve the detection capability, including photoelectrochemical assay, fluorescence assay, surface plasmon resonance assay, and surface-enhanced Raman scattering (SERS) assay. ${ }^{13-16}$

Among these techniques, SERS spectroscopy has attracted great attention and become a powerful spectroscopy technique 
due to its rapid and sensitive detection capability. ${ }^{17-19}$ The detection sensitivity of SERS is 10-14 orders of magnitude higher than that of conventional Raman spectroscopy, which is similar to that of fluorescence detection. ${ }^{18}$ Recently, a SERSbased immunosensor using functionalized nanotags has become a promising alternative for highly sensitive detection with ELISA. ${ }^{13,20-22}$ SERS nanotags are a kind of novel light scattering optical probe composed of Raman reporter molecules and noble metal nanoparticles; when they are exposed to a single excitation light source, the incident field is significantly enhanced at active sites called electromagnetic "hot spots" due to localized surface plasmon effects. ${ }^{18}$ In addition, it possesses many advantages over fluorescence dyes and quantum dots for their tremendous quantification capability, multiplexing capacity, and high photostability and biocompatibility. Up to now, SERS-based immunoassays have been applied for investigations reaching from the building blocks of life (DNA and RNA) to functional complex structures (proteins and polysaccharide) and whole cells and tissues. ${ }^{23-26}$

In the present work, we report a simple and sensitive SERSbased competitive immunosensor for OTA detection. Briefly, monoclonal anti-OTA antibody-modified magnetic beads were used as supporting substrates; the samples were added followed by OTA-BSA-immobilized SERS nanotags. After incubation and washing, the Raman signal was recorded from the magnetic bead immunocomplexes, which was found to decrease with the increasing level of OTA in the samples. Compared to planar substrates, the magnetic beads possess two main advantages. ${ }^{13,27}$ First, they have a high loading density of immunocomplexes due to their high surface-tovolume ratio. Second, the magnetic bead-based immunoassay requires a short reaction time due to its fast-molecular diffusion near three-dimensional magnetic beads. By taking advantage of the SERS nanotags and functional magnetic beads, our proposed method greatly reduces the assay time and lowers the limit of detection (LOD). In addition, this simple detection platform can be used for real-time analysis in the field using a commercialized portable Raman system. We expect that this proposed method will provide another insight into food security analysis.

\section{Experimental}

\subsection{Reagents}

Gold chloride trihydrate $\left(\mathrm{HAuCl}_{4} \cdot 3 \mathrm{H}_{2} \mathrm{O}\right)$, sodium citrate $\left(\mathrm{Na}_{3}\right.$ citrate), dihydrolipoic acid (DHLA), 1-ethyl-3-(3-dimethylaminopropyl)carbodiimide (EDC), $N$-hydroxysuccinimide (NHS), bovine serum albumin (BSA), 2-( $N$-morpholino)ethanesulfonic acid (MES), phosphate-buffered saline (PBS) ( $\mathrm{pH} 7.4$, $0.1 \mathrm{M}$ ), Tween 20, ochratoxin A (OTA), fumonisin B (FUMB), aflatoxin B1 (AFB1), and ochratoxin A-conjugated BSA (OTA-BSA) were purchased from Sigma-Aldrich (St Louis, MO, USA). Malachite green isothiocyanate (MGITC) and carboxylicactivated magnetic beads were purchased from Invitrogen Corporation (Carlsbad, CA, USA). Mouse anti-ochratoxin A monoclonal antibody (Anti-OTA antibody) was purchased from Abcam (Shanghai, China). All solutions were prepared with ultrapure water $\left(18 \mathrm{M} \Omega \mathrm{cm}^{-1}\right.$ ) obtained from a Milli-Q water purification system (Billerica, MA, USA). All reagents were of analytical reagent grade and used without further purification.

\subsection{Instrumentation}

UV-vis absorption spectra were measured by a UV-2600 UV-vis spectrophotometer (Shimadzu, Kyoto, Japan). Dynamic light scattering (DLS) data were obtained using a Nano-ZS90 apparatus (Malvern Instruments, Malvern, UK). High-magnification transmission electron microscopy (TEM) images were acquired using a JEOL JEM 2100F instrument (JEOL, Tokyo, Japan). Raman measurements were carried out using a Renishaw inVia Raman microscope system (Renishaw, UK).

\subsection{Preparation of SERS nanotag}

Gold nanoparticles (Au NPs) were synthesized according to the previously reported kinetically controlled seed-growth method by Bastús et al. ${ }^{28}$ Briefly, $75 \mathrm{~mL}$ of $2.2 \mathrm{mM} \mathrm{Na}_{3}$-citrate was heated to boiling in a three-necked round-bottomed flask with rapid magnetic stirring. As soon as boiling commenced, $0.5 \mathrm{~mL}$ of $25 \mathrm{mM} \mathrm{HAuCl}_{4}$ was added, and the mixture changed from yellow to bluish grey and then to a soft pink color within $10 \mathrm{~min}$. The resulting gold seeds were cooled to $90{ }^{\circ} \mathrm{C}$ and then $0.5 \mathrm{~mL}$ of $60 \mathrm{mM} \mathrm{Na}$-citrate and $0.5 \mathrm{~mL}$ of $25 \mathrm{mM}$ $\mathrm{HAuCl}_{4}$ were alternately added 12 times (time delay $\sim 2 \mathrm{~min}$ ). Finally, the solution was kept stirring under $90{ }^{\circ} \mathrm{C}$ for $30 \mathrm{~min}$ and then cooled to room temperature. The color of the resultant solution changed from pink to deep red. SERS nanotags were prepared by the published procedure; ${ }^{13} 5 \mu \mathrm{L}$ of $10^{-5} \mathrm{M}$ MGITC was added to $1 \mathrm{~mL}$ of $0.1 \mathrm{nM}$ Au NPs. The MGITC-Au NP mixture was reacted for $30 \mathrm{~min}$ under gentle stirring. DHLA was added as a linker and the solution was incubated for $2 \mathrm{~h}$. Then, the unreacted chemical reagents were removed by centrifugation. The $-\mathrm{COOH}$ terminal groups on the surfaces of AuNPs were activated by adding EDC and NHS. After $40 \mathrm{~min}$, OTA-BSAs were reacted with the solution for $2 \mathrm{~h}$ at room temperature. Finally, the unreacted sites on the surfaces of the Au NPs were deactivated using $1 \mathrm{mM}$ ethanolamine for $30 \mathrm{~min}$. The nonspecific binding chemicals were washed away through centrifugation twice and the remaining SERS nanotags were suspended in a PBS buffer solution ready for further use.

\subsection{Preparation of antibody-conjugated magnetic bead}

To conjugate anti-OTA antibodies on the surface of magnetic beads, $5 \mu \mathrm{L}$ of $0.1 \mathrm{M}$ EDC and NHS were added to $400 \mu \mathrm{L}$ of $0.5 \mathrm{mg} \mathrm{mL} \mathrm{m}^{-1}$-COOH terminal group-modified magnetic beads and the mixture was incubated with gentle shaking at room temperature. After $30 \mathrm{~min}$, the mixture was separated by a magnet and washed three times with PBS buffer solution. Subsequently, the magnetic beads were resuspended in PBS buffer solution and reacted with $1 \mathrm{mg} \mathrm{mL}^{-1}$ anti-OTA antibodies overnight under continuous shaking. The unreacted $-\mathrm{COOH}$ groups were deactivated by incubation with $1 \%$ BSA 
for 30 min. Finally, the above mixture was washed with a PBS buffer solution to remove unbound chemicals and stored in PBS buffer solution at $4{ }^{\circ} \mathrm{C}$ before use.

\subsection{SERS-based competitive immunoassay for OTA}

Competitive immunoassay for OTA was performed with spiked samples at nine different concentrations. Briefly, $20 \mu \mathrm{L}$ of antibody-conjugated magnetic beads, $10 \mu \mathrm{L}$ of OTAspiked sample and $40 \mu \mathrm{L}$ of SERS nanotags were mixed and allowed to react under constant stirring for $1 \mathrm{~h}$. The mixture was isolated by a magnetic bar on the wall of the microtube and washed three times with PBS containing $0.05 \mathrm{wt} \%$ Tween 20. Finally, the immunocomplexes were resuspended in PBS buffer solution and transferred to a capillary tube for Raman analysis.

\subsection{Preparation of wine samples}

To evaluate the practicality of the developed sensing method, wine obtained from the local supermarket was diluted 5 times with PBS buffered solution, and then passed through a $0.22 \mu \mathrm{m}$ membrane. The OTA stock solution was spiked in the above purified wine to prepare $10 \mathrm{pg} \mathrm{mL}^{-1}, 100 \mathrm{pg} \mathrm{mL}^{-1}$, and $500 \mathrm{pg} \mathrm{mL}{ }^{-1}$ OTA wine samples.
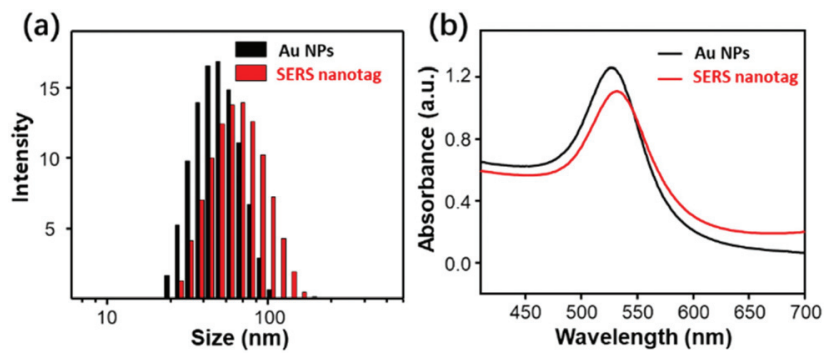

Fig. 1 (a) DLS distributions of AuNPs and SERS nanotags; (b) UV-vis spectra of Au NPs and SERS nanotags.

\section{Results and discussion}

\subsection{Principle of SERS-based competitive immunoassay for detection of OTA}

The SERS nanotag consists of Au NPs, Raman reporters, and OTA-BSA conjugates. The Au NPs were synthesised by the seed growth method. MGITC was used as the Raman reporter and the strongest peak intensity that centered at $1613 \mathrm{~cm}^{-1}$ was used for the quantitative analysis of OTA. DHLA bonded with the $\mathrm{Au}$ NP surface through the interactions of Au atoms with the thiol group. The $-\mathrm{COOH}$ groups were activated by EDC and NHS, with the OTA-BSA conjugates subsequently being immobilized on Au NPs. The effects of OTA-BSA conjugates immobilized on the surface of Au NPs were assessed by UV-vis and DLS measurements. The average hydrodynamic size of the Au NPs increased from 44 to $53 \mathrm{~nm}$ upon conjugation, according to the DLS data shown in Fig. 1a. The UV-vis spectral result also demonstrated that the surface plasmon band was slightly shifted from $527 \mathrm{~nm}$ to $532 \mathrm{~nm}$ as shown in Fig. 1b.

Scheme 1 shows a schematic of the SERS-based competitive immunoassay. Here, the magnetic beads were employed as the separation and concentration agents, and the Au NPs were employed as the SERS substrate for signal amplification. As shown in Fig. 2a, in the absence of target OTA, the SERS nanotags are captured by anti-OTA antibodies on the surface of the magnetic beads. These magnetic immunocomplexes were washed and isolated using a magnetic bar. Strong SERS signals were observed due to the SERS effect of the SERS nanotags upon the exposure of the laser, as seen in Fig. 2c. However, in the presence of target OTA, a competitive reaction toward anti-OTA immobilized magnetic beads occurs between the target OTA and SERS nanotags. When the anti-OTA antibodies capture target OTA, they cannot react with the SERS nanotag.s. Consequently, as shown in Fig. 2b, the number of SERS nanotags bound on magnetic beads decreased, which resulted in the very low SERS signal, as seen in Fig. 2c.

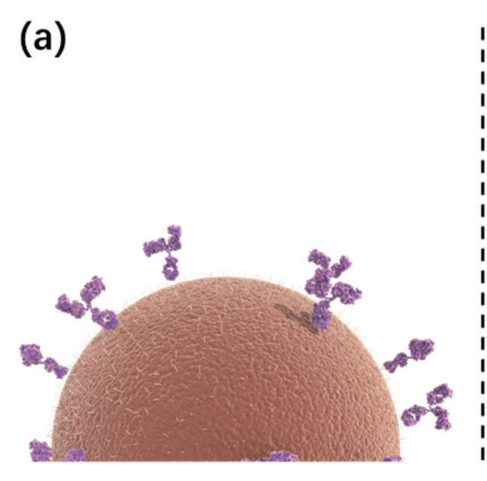

Magnetic beads
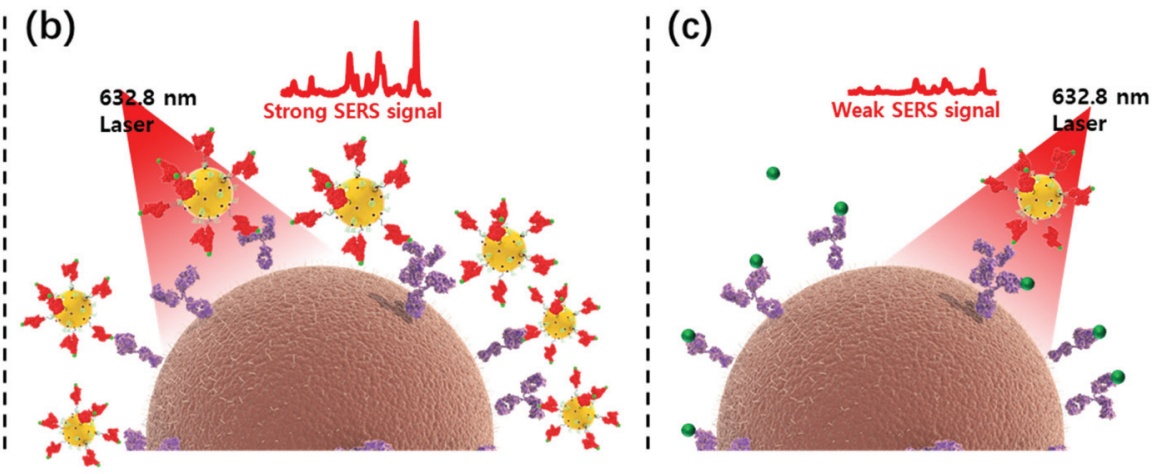

SERS nanotag

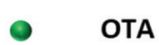

Scheme 1 Schematic illustration of the SERS-based competitive immunoassay for the determination of OTA. (a) Anti-OTA antibody immobilized on the magnetic beads; (b) OTA-BSA-conjugated SERS nanotags were captured by the anti-OTA antibodies on the magnetic beads; (c) target OTA and OTA-BSA conjugated SERS nanotags competitively react with anti-OTA antibody on the magnetic beads. 
(a)

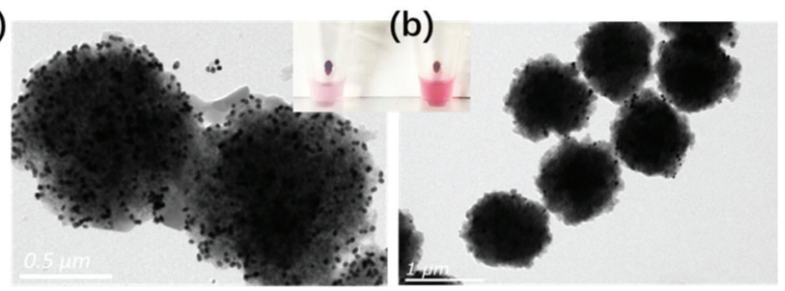

(c)

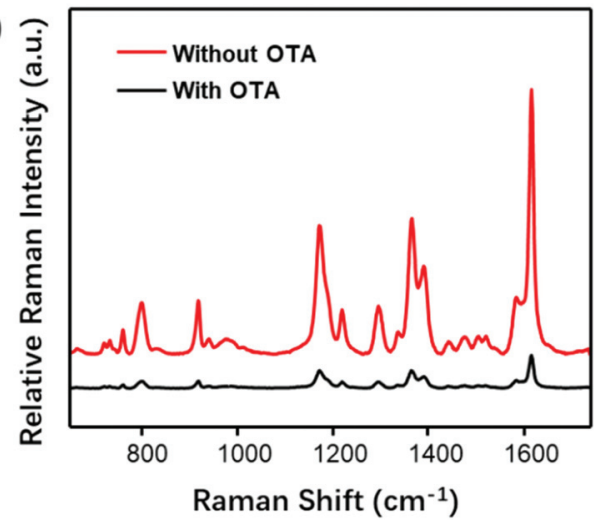

Fig. 2 SEM images of the SERS-based competitive immunoassay without (a) and with (b) target OTA; (c) corresponding SERS spectra of (a) and (b).

Thus, in this assay, the SERS signal decreased with the increasing level of target OTA in the sample, and the SERS signal of the formed immunocomplexes can be measured and analysed for the quantitative evaluation of target OTA.

\subsection{Optimization of immunoassay parameters}

To acquire an optimal analytical condition for the SERS-based competitive assay, the volume ratio of magnetic beads to SERS nanotags and the incubation time for the competitive immunoassay were systematically investigated. The optimization process was carried out by changing one parameter while keeping all the others constant.

The volume ratio of magnetic beads to SERS nanotags was very crucial since it strongly affected the detection sensitivity of the competitive immunoassay. Therefore, the volume ratio of magnetic beads to SERS nanotags $(1: 0.5,1: 1,1: 1.5,1: 2$, $1: 2.5,1: 3$, and $1: 4$ ) was first explored. Specifically, $20 \mu \mathrm{L}$ of magnetic beads were incubated with different volumes of SERS nanotags for $2 \mathrm{~h}$, followed by washing three times with PBS-Tween 20 (PBST), and dispersing in PBS. As shown in Fig. 3a, the Raman intensity gradually increased with increase in the volume of SERS nanotags in the range of $1: 0.5$ to $1: 2$. However, a further increase in the volume of SERS nanotags could not cause significant differences in Raman intensity because as the anti-OTA antibodies on the surfaces of magnetic beads were fully occupied by the SERS nanotags, no binding sites were remaining on the surface of magnetic beads for the excess SERS nanotags. Based on this result, the optimal volume ratio was determined to be $1: 2$ in this work.

Next, we investigated the incubation time of the anti-OTA immobilized magnetic beads and SERS nanotags for the detec- (a)

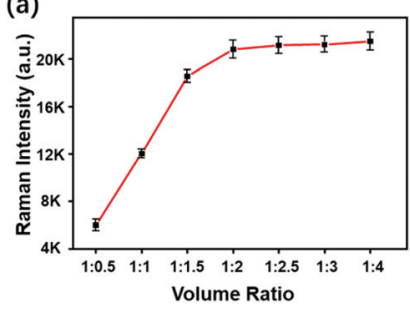

(b)

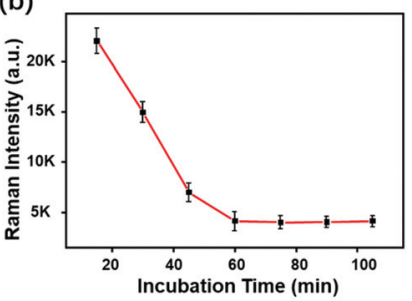

Fig. 3 Optimization of immunoassay parameters. (a) Volumetric ratio of magnetic beads and SERS nanotags; and (b) incubation time for the competitive reaction among magnetic beads, SERS nanotags, and target OTA.

tion of OTA. Specifically, $20 \mu \mathrm{L}$ of magnetic beads, $40 \mu \mathrm{L}$ of SERS nanotags and $10 \mu \mathrm{L}$ of OTA were incubated for $15 \mathrm{~min}$, $30 \mathrm{~min}, 45 \mathrm{~min}, 60 \mathrm{~min}, 75 \mathrm{~min}, 90 \mathrm{~min}$, and $105 \mathrm{~min}$, respectively. As shown in Fig. 3b, the Raman intensity decreased gradually with the incubation time increasing up to $60 \mathrm{~min}$ and further incubation did not cause any significant change. Our results indicate that the competitive reaction was complete within $60 \mathrm{~min}$. Therefore, $60 \mathrm{~min}$ was selected as the optimal incubation time in the subsequent assays.

\subsection{Analytical performance of the SERS-based immunoassay}

Under optimized detection conditions, the performance of the SERS-based competitive detection platform for the quantitative analysis of OTA was evaluated. Fig. 4a shows the Raman spectra for various concentrations of OTA in the 0 to $2500 \mathrm{pg}$ $\mathrm{mL}^{-1}$ range, which was in accordance with the fact that with an increase in the OTA concentration, more targets were able to form immunocomplexes with the magnetic beads and fewer SERS nanotags would bind to the magnetic beads. Thus, the Raman intensity was gradually decreased. The corresponding calibration plot from the Raman intensity variations at $1613 \mathrm{~cm}^{-1}$ is shown in Fig. 4b and the error bars indicate standard deviations from three measurements. The limit of detection (LOD) value was calculated to be $0.61 \mathrm{pg} \mathrm{mL}^{-1}$ by the IUPAC standard method (LOD $=y_{\text {blank }}+3 \times$ SD blank $_{\text {blank }}$ is the average signal intensity at zero and $\mathrm{SD}_{\text {blank }}$ is the standard deviation of the blank measurements). The value determined

(a)

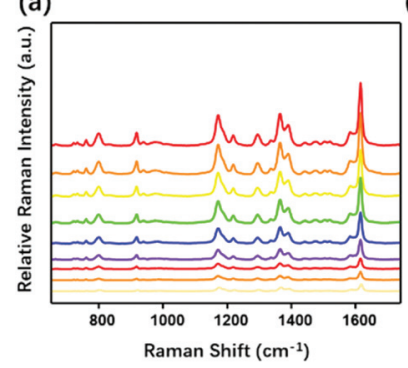

(b)

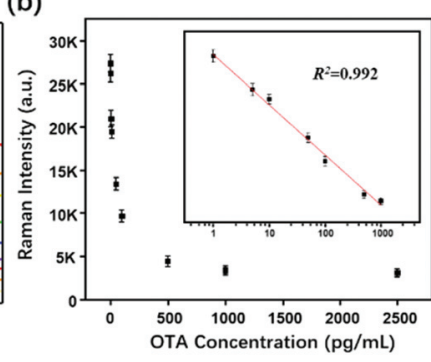

Fig. 4 (a) SERS spectra of the increasing concentrations of OTA ranging from 0 to $2500 \mathrm{pg} \mathrm{mL}^{-1}$; and (b) variation of SERS signal at $1613 \mathrm{~cm}^{-1}$ as a function of OTA concentration. The inset shows a linear relationship in the concentration range from $1 \mathrm{pg} \mathrm{mL}^{-1}$ to $1000 \mathrm{pg} \mathrm{mL}^{-1}$. 
Table 1 Comparison of the SERS-based competitive assay results with the previously reported methods using other detection techniques

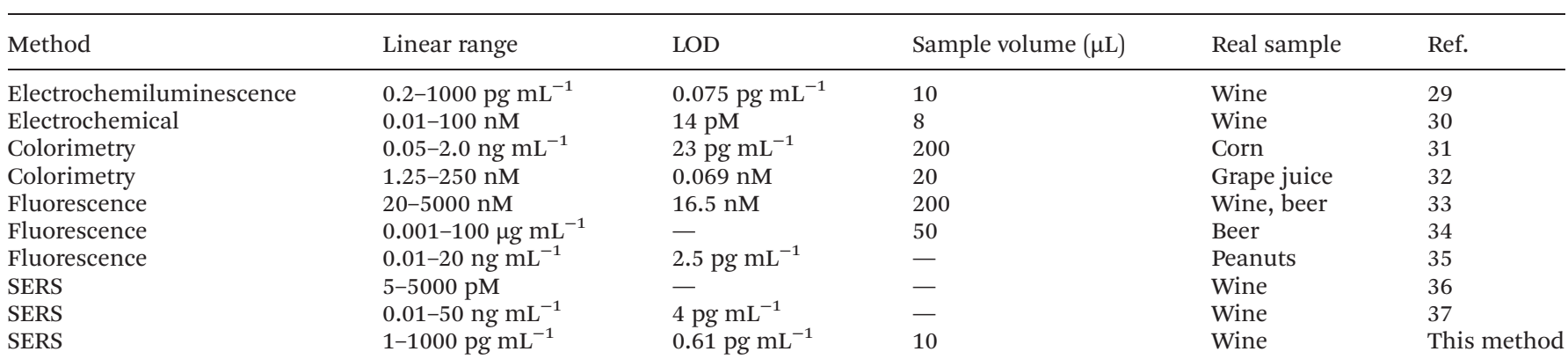

by the proposed method is more sensitive than that of the traditional ELISA method and the reported methods for OTA detection as shown in Table 1.

\subsection{Specificity of the SERS-based immunoassay}

The specificity of the SERS-based assay platform was also investigated. Two mycotoxins, zearalenone (ZEN) and aflatoxin B1 (AFB1) were selected as the negative controls because they are very commonly found mycotoxins in food and environment; ${ }^{38,39}$ their chemical structures are shown in Fig. 5a. The tests were performed for five samples with different mycotoxins. Sample 1 contains $0 \mathrm{pg} \mathrm{mL}^{-1}$ myco-

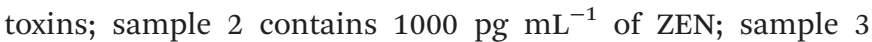
contains $1000 \mathrm{pg} \mathrm{mL} \mathrm{m}^{-1}$ of AFB1; sample 4 contains 1000 pg $\mathrm{mL}^{-1}$ of OTA; and sample 5 contains $1000 \mathrm{pg} \mathrm{mL}^{-1}$ of ZEN, $1000 \mathrm{pg} \mathrm{mL} \mathrm{m}^{-1}$ of AFB1 and $1000 \mathrm{pg} \mathrm{mL}^{-1}$ of OTA. As shown in Fig. 5b and c, only OTA and its mixtures lead to a significant decrease in Raman intensity and no obvious changes were observed for the other samples. The results demonstrated that the proposed method shows high specificity for OTA.

\subsection{Real sample analysis}

Wine has been identified as the second major source of OTA ingestion by humans in Europe. To assess the practical applicability of the SERS-based competitive immunoassay, the
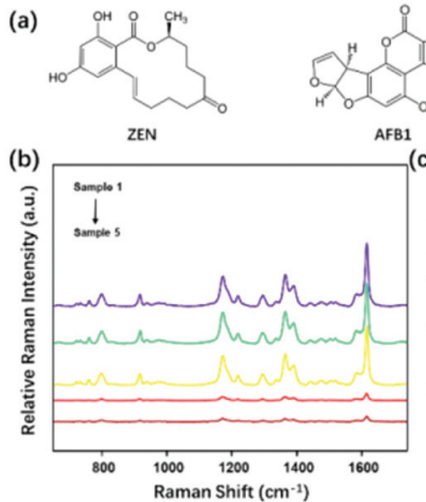

Fig. 5 (a) Molecular structures of ZEN, AFB1, and OTA; (b) SERS spectra of blank, ZEN, AFB1, OTA, and their mixtures; (c) comparison of the relative SERS signals at $1613 \mathrm{~cm}^{-1}$.
Table 2 Recovery values corresponding to four wine samples

\begin{tabular}{lllll}
\hline Sample & $\begin{array}{l}\text { Spiked } \\
\left(\mathrm{pg} \mathrm{mL} \mathrm{m}^{-1}\right)\end{array}$ & $\begin{array}{l}\text { SERS method } \\
\left(\mathrm{pg} \mathrm{mL} \mathrm{mL}^{-1}\right)\end{array}$ & $\begin{array}{l}\text { Recovery } \\
(\%)\end{array}$ & $\begin{array}{l}\text { RSD } \\
(\%)\end{array}$ \\
\hline 1 & 0 & - & - & - \\
2 & 10 & 9.06 & 90.60 & 2.97 \\
3 & 100 & 103.29 & 103.3 & 2.65 \\
4 & 500 & 492.32 & 98.46 & 2.47
\end{tabular}

developed method was applied to detect OTA in wine samples using the standard addition method. As shown in Table 2, the recovery rate of spiked wine ranged from $90.60 \%$ to $103.3 \%$, and the relative standard deviation (RSD) was between $2.47 \%$ and $2.97 \%$. The results were statistically close to the spiked value, which demonstrated that the proposed method could be successfully applied to detect OTA in wine samples.

\section{Conclusions}

In the present work, a SERS-based competitive immunoassay platform using OTA-BSA conjugated SERS nanotags and antiOTA functionalized magnetic beads was reported for the highly sensitive and specific detection of OTA. Here, the target OTA and OTA-BSA-conjugated SERS nanotags reacted competitively with the anti-OTA antibodies immobilized on the surface of magnetic beads. The Raman peak of the SERS nanotags at $1613 \mathrm{~cm}^{-1}$ was monitored and its variation was used for the quantitative determination of OTA. A linear relationship was obtained with a concentration range of $1 \mathrm{pg} \mathrm{mL}^{-1}$ to $1000 \mathrm{pg}$ $\mathrm{mL}^{-1}$ and the LOD was determined to be $0.61 \mathrm{pg} \mathrm{mL}^{-1}$ based on the IUPAC standard method. In addition, the SERS-based competitive assay was successfully used for detecting OTA in wine samples with a satisfying recovery of $90.60 \%-103.3 \%$. It is believed that this SERS-based competitive assay has great potential for the highly sensitive and specific quantification of various mycotoxins and other biotarget molecules.

\section{Conflicts of interest}

There are no conflicts to declare. 


\section{Acknowledgements}

This work was financially supported by grants from the National Natural Science Foundation of China (21904078 and 21976209) and the Taishan Scholar Project Special Funding (no. ts20190962).

\section{Notes and references}

1 C. L. Gerez, A. Dallagnol, L. Ponsone, S. Chulze and G. Font de Valdez, Food Control, 2014, 45, 115-119.

2 A. El Khoury and A. Atoui, Toxins, 2010, 2, 461-493.

3 T. Kuiper-Goodman and P. M. Scott, Regul. Toxicol. Pharmacol., 1987, 7, 253-306.

4 S. Amézqueta, E. González-Peñas, M. Murillo-Arbizu and A. López de Cerain, Food Control, 2009, 20, 326-333.

5 M. Rocha, F. Freire, F. Maia, M. I. Florindo Guedes and D. Rondina, Food Control, 2014, 36, 159-165.

6 A. Pfohl-Leszkowicz and R. A. Manderville, Mol. Nutr. Food Res., 2007, 51, 61-99.

7 S. C. Duarte, A. Pena and C. M. Lino, Crit. Rev. Toxicol., 2011, 41, 187-212.

8 D. Jornet, O. Busto and J. Guasch, J. Chromatogr. A, 2000, 882, 29-35.

9 W. Zhu, C. Ren, Y. Nie and Y. Xu, Food Control, 2016, 64, 37-44.

10 C. Jiang, L. Lan, Y. Yao, F. Zhao and J. Ping, TrAC, Trends Anal. Chem., 2018, 102, 236-249.

11 S. Hickert, J. Gerding, E. Ncube, F. Hübner, B. Flett, B. Cramer and H.-U. Humpf, Mycotoxin Res., 2015, 31, 109115.

12 E. P. Meulenberg, Toxins, 2012, 4, 244-266.

13 X. Wang, S.-G. Park, J. Ko, X. Xiao, V. Giannini, S. A. Maier, D.-H. Kim and J. Choo, Small, 2018, 14, 1801623.

14 H. Jiang, X. Li, Y. Xiong, K. Pei, L. Nie and Y. Xiong, Toxins, 2017, 9, 83.

15 A. Karczmarczyk, C. Reiner-Rozman, S. Hageneder, M. Dubiak-Szepietowska, J. Dostálek and K.-H. Feller, Anal. Chim. Acta, 2016, 937, 143-150.

16 Y. Li, N. Zhang, H. Wang and Q. Zhao, Analyst, 2020, 145, 651-655.

17 Z. Wang, S. Zong, L. Wu, D. Zhu and Y. Cui, Chem. Rev., 2017, 117, 7910-7963.

18 Y. Wang, B. Yan and L. Chen, Chem. Rev., 2013, 113, 13911428.
19 C. Zong, M. Xu, L.-J. Xu, T. Wei, X. Ma, X.-S. Zheng, R. Hu and B. Ren, Chem. Rev., 2018, 118, 4946-4980.

20 Z. Cheng, N. Choi, R. Wang, S. Lee, K. C. Moon, S.-Y. Yoon, L. Chen and J. Choo, ACS Nano, 2017, 11, 4926-4933.

21 R. Wang, H. Chon, S. Lee, Z. Cheng, S. H. Hong, Y. H. Yoon and J. Choo, ACS Appl. Mater. Interfaces, 2016, 8, 1066510672.

22 M. D. Porter, R. J. Lipert, L. M. Siperko, G. Wang and R. Narayanan, Chem. Soc. Rev., 2008, 37, 1001-1011.

23 S. H. Lee, J. Hwang, K. Kim, J. Jeon, S. Lee, J. Ko, J. Lee, M. Kang, D. R. Chung and J. Choo, Anal. Chem., 2019, 91, 12275-12282.

24 Y. Wu, N. Choi, H. Chen, H. Dang, L. Chen and J. Choo, Anal. Chem., 2020, 92, 2628-2634.

25 X. Wang, N. Choi, Z. Cheng, J. Ko, L. Chen and J. Choo, Anal. Chem., 2017, 89, 1163-1169.

26 J. Su, D. Wang, L. Nörbel, J. Shen, Z. Zhao, Y. Dou, T. Peng, J. Shi, S. Mathur, C. Fan and S. Song, Anal. Chem., 2017, 89, 2531-2538.

27 A. Tao, F. Kim, C. Hess, J. Goldberger, R. He, Y. Sun, Y. Xia and P. Yang, Nano Lett., 2003, 3, 1229-1233.

28 N. G. Bastús, J. Comenge and V. Puntes, Langmuir, 2011, 27, 11098-11105.

29 Y. Chen, M. Yang, Y. Xiang, R. Yuan and Y. Chai, Nanoscale, 2014, 6, 1099-1104.

30 L. Rivas, C. C. Mayorga-Martinez, D. Quesada-González, A. Zamora-Gálvez, A. de la Escosura-Muñiz and A. Merkoçi, Anal. Chem., 2015, 87, 5167-5172.

31 C. Lin, H. Zheng, M. Sun, Y. Guo, F. Luo, L. Guo, B. Qiu, Z. Lin and G. Chen, Anal. Chim. Acta, 2018, 1002, 90-96.

32 F. Tian, J. Zhou, B. Jiao and Y. He, Nanoscale, 2019, 11, 9547-9555.

33 L. Lv, D. Li, R. Liu, C. Cui and Z. Guo, Sens. Actuators, B, 2017, 246, 647-652.

34 Z. Lv, A. Chen, J. Liu, Z. Guan, Y. Zhou, S. Xu, S. Yang and C. Li, PLoS One, 2014, 9, e85968-e85968.

35 J. Tian, W. Wei, J. Wang, S. Ji, G. Chen and J. Lu, Anal. Chim. Acta, 2018, 1000, 265-272.

36 X. Jing, L. Chang, L. Shi, X. Liu, Y. Zhao and W. Zhang, ACS Appl. Bio Mater., 2020, 3, 2385-2391.

37 B. Shao, X. Ma, S. Zhao, Y. Lv, X. Hun, H. Wang and Z. Wang, Anal. Chim. Acta, 2018, 1033, 165-172.

38 Y. Liu, C.-C. H. Chang, G. M. Marsh and F. Wu, Eur. J. Cancer, 2012, 48, 2125-2136.

39 A. Zinedine, J. M. Soriano, J. C. Moltó and J. Mañes, Food Chem. Toxicol., 2007, 45, 1-18. 